\title{
Promoting Scale-Up Across a Global Project Platform: Lessons from the Evidence to Action Project
}

\author{
Laura Ghiron $^{1}$ (D) $\cdot$ Eric Ramirez-Ferrero ${ }^{2} \cdot$ Rita Badiani $^{2} \cdot$ Regina Benevides $^{3} \cdot$ Alexis Ntabona $^{4} \cdot$ Peter Fajans $^{5}$. \\ Ruth Simmons ${ }^{6}$
}

Received: 9 August 2020 / Accepted: 28 April 2021 / Published online: 18 May 2021

(c) The Author(s) 2021

\begin{abstract}
The USAID-funded flagship family planning service delivery project named Evidence to Action (E2A) worked from 2011 to 2021 to improve family planning and reproductive health for women and girls across seventeen nations in sub-Saharan Africa using a "scaling-up mindset." The paper discusses three key lessons emerging from the project's experience with applying ExpandNet's systematic approach to scale up. The methodology uses ExpandNet/WHO's scaling-up framework and guidance tools to design and implement pilot or demonstration projects in ways that look ahead to their future scaleup; develop a scaling-up strategy with local stakeholders; and then strategically manage the scaling-up process. The paper describes how a scaling-up mindset was engendered, first within the project's technical team in Washington and then how they subsequently sought to build capacity at the country level to support scale-up work throughout E2A's portfolio of activities. The project worked with local multi-stakeholder resource teams, often led by government officials, to equip them to lead the scale-up of family planning and health system strengthening interventions. Examples from project experience in the Democratic Republic of the Congo, Kenya, Nigeria, and Uganda illustrating key concepts are discussed. E2A also established a community of practice on systematic approaches to scale up as a platform for sharing learning across a variety of technical agencies engaged in scale-up work and to create learning opportunities for interacting with thought leaders around critical scale-up issues.
\end{abstract}

Keywords Scaling up framework · Implementation $\cdot$ ExpandNet $\cdot$ E2A $\cdot$ Family planning $\cdot$ Capacity building $\cdot$ Country ownership - Mindset

\section{Introduction}

Laura Ghiron

ljghiron@umich.edu

1 ExpandNet Secretariat and Partners in Expanding Health Quality and Access, Davis, CA, USA

2 Pathfinder International/Evidence to Action (E2A) Project, Washington, DC, USA

3 Pathfinder International/E2A Project (2011-2018), Atlanta, GA, USA

4 ExpandNet, Kinshasa, Democratic Republic of the Congo

5 ExpandNet Secretariat, Gex, France

6 University of Michigan School of Public Health and ExpandNet Secretariat, Inverness, CA, USA

Several decades of investment by global donors to improve health and other areas of development have not succeeded in ensuring that interventions proven on a small scale become part of national systems (Simmons et al., 2007; Hartmann \& Linn, 2008; ExpandNet \& World Health Organization, 2009). More recently, there have been increasing calls to design and implement interventions using a scale-up perspective (ExpandNet \& World Health Organization, 2009, 2010, 2011; Barker et al., 2016) that gives explicit attention to "increasing the impact of successfully tested interventions so as to benefit more people and to foster policy and program development on a lasting basis" (Simmons et al., 2007). Many publications in the last twelve years have assessed the determinants of scale-up success and laid out frameworks and practical guidance to support the scale-up process (Simmons et al., 2007; Hartmann \& Linn, 2008; 
ExpandNet \& World Health Organization, 2009, 2010, 2011; Yamey, 2011; Chandy \& Linn, 2011; Chandy et al., 2013; Cooley \& Linn, 2014; Milat et al., 2015; Barker et al., 2016; Milat et al., 2016; Management Systems International, 2016; Spicer et al., 2018; Bulthuis et al., 2020). Other publications have focused on sharing in-country project experience of scale-up activities (Ghiron et al., 2014; Igras et al., 2014; Spicer et al., 2014; Keyonzo et al., 2015; Aichatou et al., 2016; Fajans \& Simmons, 2016; Omimo et al., 2018; Mai et al., 2019; Benevides et al., 2019; Oku, 2019; ExpandNet, 2021). These have all contributed to shifting the global health and development paradigm away from more conventional project approaches (Keyonzo et al., 2015) to ones that use knowledge about what works for scale-up to shape the introduction of new interventions and their subsequent expansion and institutionalization. However, there has been little documentation of how large multi-country, donorfunded projects have sought to address the importance of scale-up in the design and implementation of their initiatives (Simmons et al., 2007; Chandy et al., 2013; Barker et al., 2016; Fajans \& Simmons, 2016).

This paper highlights how USAID's global flagship family planning and reproductive health $(\mathrm{FP} / \mathrm{RH})$ project, "Evidence to Action" (E2A), (E2A, 2020) worked with ExpandNet over nine years to implement a scale-up focus in its activities. Prepared by members of ExpandNet and the E2A project's technical leadership team, the paper provides key learning from this process with the hope that other initiatives can benefit and in turn help countries more rapidly achieve their ambitious Sustainable Development Goals targets and commitments (United Nations, 2015). The authors played different roles in the project. The E2A team leaders were full-time employees responsible for all of the project's activities at the global and country levels, whereas ExpandNet members functioned as part-time consultants providing support to the project at the global level and in select countries on the topic of scale-up, but not for other aspects of the technical work. All authors were participant observers in the change processes described in the paper. A narrative presentation was chosen to convey the rich story of how capacity building on the determinants of successful scale-up was achieved among collaborators at the project headquarters and country levels.

\section{ExpandNet's Systematic Approach to Scaling Up}

Founded in 2003, with leadership from the University of Michigan School of Public Health and the World Health Organization (WHO) Department of Reproductive Health and Research, ExpandNet is a global network of public health and development professionals who seek to advance the science and practice of scale-up. Based on ExpandNet members' practical experience and extensive reviews of literature from a range of fields and disciplines, ExpandNet and WHO jointly published a book presenting a scalingup framework and seven country case studies of experience (Simmons et al., 2007). Subsequently, in an effort to support scale-up practitioners, ExpandNet and WHO published three practical guidance tools, all of which are available on both the ExpandNet and WHO websites (ExpandNet \& WHO, 2009, 2010, 2011; ExpandNet, 2021). The ExpandNet/WHO framework and tools provide a "roadmap" to help public health and development practitioners use critical lessons about scale-up to support a process whereby interventions tested on a small scale are expanded and institutionalized to reach more people on a sustainable basis.

Applying the ExpandNet/WHO scaling-up framework and guidance tools is part of ExpandNet's systematic approach to scale up, which is divided into three phases, as depicted in Fig. 1 (Omimo et al., 2018; ExpandNet,
Fig. 1 ExpandNet's systematic approach to scale up, adapted from Omimo et al. (2018)

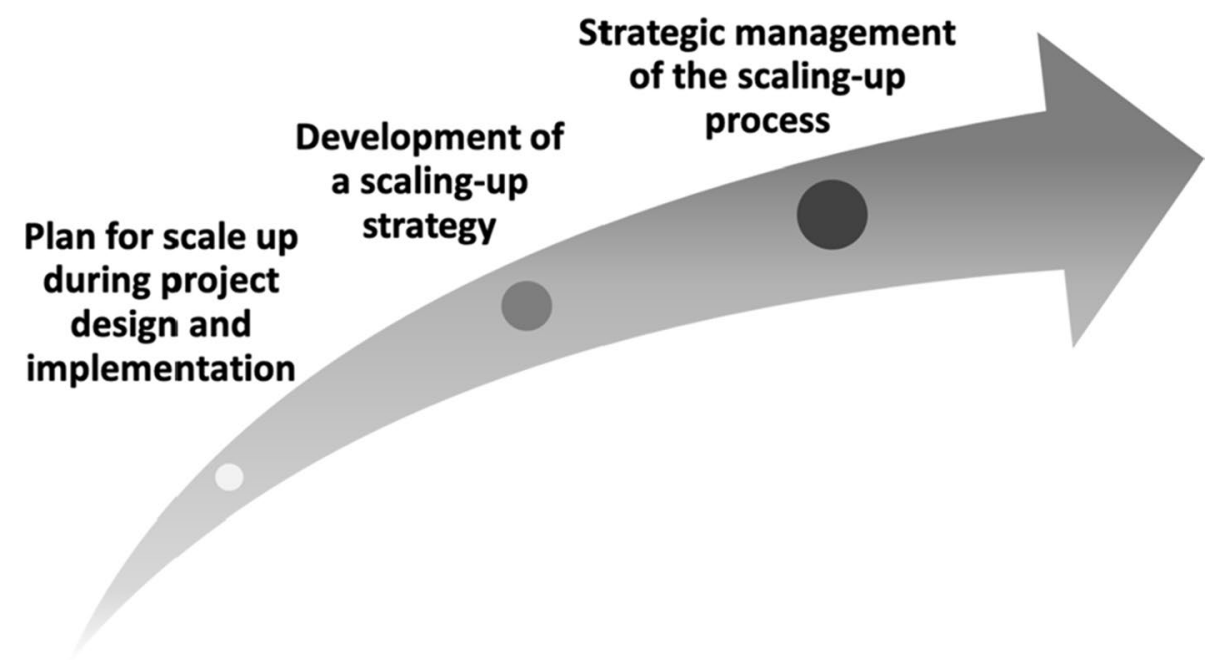


2021). Phase 1 , focused on testing interventions, uses learning about scale-up success to shape a project's design and implementation; Phase 2 supports systematically developing a scale-up strategy, once results of local testing indicate this is appropriate; and Phase 3 is about strategically managing the scaling-up process. Since 2007, ExpandNet and many organizations and individuals have been using the ExpandNet/WHO framework and approach to provide technical support on scale-up to a broad range of activities in low- and middle-income countries along the three phases illustrated in Fig. 1 (Ghiron et al., 2014; Igras et al., 2014; Keyonzo et al., 2015; Fajans \& Simmons, 2016; Aichatou et al., 2016; Omimo et al., 2018; Benevides et al., 2019; Mai et al., 2019; Oku, 2019; ExpandNet, 2021).

\section{The Evidence to Action Project}

The 2011 USAID solicitation for the Evidence to Action project mandated a scale-up focus for the new family planning (FP) service delivery project. Pathfinder International's winning proposal was developed collaboratively with ExpandNet and the three other core partners-IntraHealth, Management Sciences International, and PATH. It emphasized that the project would support governments and local development partners to introduce promising and proven FP best practices, including those globally agreed to be FP High-Impact Practices, with scale-up in mind and to facilitate their expansion and institutionalization. The strong emphasis on government ownership was based on the understanding that unless the intended adopters of interventions (e.g. ministries of health) consider donor-funded interventions a priority and feasible for implementation within their institutions, they are unlikely to be sustainably scaled (Simmons et al., 2007; ExpandNet \& WHO, 2010, 2011; Yamey, 2011; Milat et al., 2015; Milat et al., 2016; Spicer et al., 2018; Wickremasinghe et al., 2018; Bulthuis et al., 2020).

Since E2A began, its mandate has been to help introduce or scale up best practices in family planning and reproductive health $(\mathrm{RH})$ service delivery for women and girls. In addition to providing global thought leadership, the project has worked across seventeen countries in Francophone and Anglophone sub-Saharan Africa. The range of project activities included improving quality of care in the provision of FP/RH services for adolescents, youth and first-time parents, expanding contraceptive method choice and integrating family planning with other health and development interventions.

While E2A's country and regional activities differed, nearly all maintained an explicit, ongoing focus on using global lessons about scale-up to shape interventions for implementation within the intended institutions. This focus is here referred to as using a "scaling-up mindset" that recognizes the importance of (a) applying a systems perspective that focuses on the broader national program when designing and implementing interventions, (b) ensuring government and other local stakeholders' ownership of the initiative, (c) making certain that activities/investments contribute meaningfully to the national program, and (d) limiting external inputs to what can be maintained or mobilized when implementing on a larger scale.

Such a systems-strengthening approach stands in contrast to the classic "project approach," defined by Keyonzo et al. (2015, p. 104) as "interventions that are focused on shortterm results and not necessarily on what is needed to ensure sustainable scale-up, even though large-scale impact is often stated as the ultimate goal. In this approach levels of financial, technical and human resources that are unlikely to be available for subsequent implementation in routine programs and parallel structures to existing government or private sector programs tend to be created."

With support from ExpandNet members, E2A's application of the ExpandNet framework and tools enabled the project to apply a scaling-up mindset and have a common language and unifying approach to the supported country initiatives. Achieving the shift to a scaling-up mindset took time and continuous attention. Three overarching lessons from this experience are discussed below.

\section{Lessons from Applying a Systematic Scale-Up Approach}

\section{Lesson 1: A Systematic Approach to Scale-Up Requires Shifting Mindsets Followed by Capacity Building}

If development interventions are to be successfully expanded and institutionalized in country settings, it is critical that technical staff supporting such processes have sufficient expertise to ensure their activities are carried out using a scaling-up mindset (ExpandNet \& WHO, 2010; Milat et al., 2016; Keyonzo et al., 2015). E2A's proposal development team had embraced the ExpandNet systematic approach and discussed in the proposal how it would be applied across countries. However, implementers, technical partners, governments, other NGOs, and donors with whom E2A would eventually work in countries were not necessarily accustomed to the processes being championed by E2A, which sometimes represented a significant departure from customary local work patterns and models of technical assistance.

Even the newly constituted headquarters' nine-person implementation team in Washington DC (hereafter referred to as E2A's technical team) was initially unfamiliar with developments in the scale-up field. Thus, a key first step was for them to gain exposure to ExpandNet's systematic 
approach and related tools and to begin to shift away from conventional project approaches. In support, ExpandNet provided a series of half-day seminars over a few-months period - with roughly 15 hours total contact time - to expose the technical team to scaling-up principles presented in the tools and key lessons from ExpandNet's prior use of them with country projects.

Despite being helpful, the early seminars were not sufficient to fully equip the technical team to support application of the ExpandNet/WHO approach at the country level. Therefore, ExpandNet members continued over the next few years to collaborate with technical team members to bolster their capacity through mentoring and jointly facilitating country-level planning exercises, delivering scale-up workshops, and supporting scale-up analysis and documentation efforts. This hands-on support to the individual team members who were supporting specific E2A country initiatives helped them to internalize the scale-up framework and key principles, to critically analyze strategic issues and better support countries to move forward with their scale-up efforts. As in any team, a few members of the technical team were more responsive to the ideas being shared and able to rapidly assimilate them into their work with countries. ExpandNet members ultimately worked more intensively with these project champions of the scale-up approach and over time these individuals required decreasing levels of collaboration from ExpandNet to achieve results.

As highlighted under lesson two below, with this new mindset and growing scale-up capacity, the E2A team began to see significant impact in shifting country-level activities from conventional project approaches to a focus on scaling up. In several instances, country leaders expressed that this new way of working constituted a productive and valuable departure from more traditional project approaches. Nonetheless, sometimes the change in mindset among country colleagues was hard to achieve, and in some places, it did not succeed. This was most often the case where E2A's role was limited or peripheral, for example, when an existing project was already approved and underway by the time E2A was invited to collaborate.
ExpandNet's experience over the years has shown that commitment to engage in systematic scale-up planning works best when country ownership is prioritized from the earliest stages of the project planning process. Likewise, scaling-up capacity is best built when technical assistance is provided in the context of real-time applied scale-up work. In E2A project countries where long-time ExpandNet members were based and could be mobilized to give local/regional support to E2A scale-up activities, as in the Democratic Republic of the Congo (Mai et al., 2019) and Senegal, the work benefited greatly.

\section{Lesson 2: A Country Resource Team With Scale-Up Capacity can Accomplish Much, but Challenges Remain}

As E2A's technical team in Washington DC began supporting work in countries, a priority was to identify and build capacity of a local "resource team" of stakeholders who would own, lead, and support scale-up processes (Simmons et al., 2007; ExpandNet \& WHO, 2009, 2010). ExpandNet/ WHO defines a resource team as "the individuals and organizations that seek to promote and facilitate wider use of the innovation. A resource team may be formally charged with promoting the innovation or may act informally in this role." (ExpandNet \& WHO, 2010, p. 6). Key attributes of resource teams that contribute to scale-up success are highlighted in Table 1.

Applications of the ExpandNet approach in several countries has shown that when scale-up was intended to take place within government systems, as was often the case in E2A activities, it was critical that government played a leading role in the resource team (Simmons et al., 2007; Keyonzo et al., 2015; Mai et al., 2019; Benevides et al., 2019; Oku et al., 2019). Such leadership builds government ownership of scale-up activities, even when efforts are being supported by technical assistance agencies. It also prevents the initiative from being perceived as an NGO- or donor-led effort, which can negatively impact local ownership, institutionalization of interventions, and resource mobilization. The resource team should ideally
Table 1 Key attributes of a successful scale-up resource team (Simmons et al., 2007; ExpandNet \& World Health Organization, 2009, 2010)

\author{
Effective leadership with authority and credibility \\ A unifying vision and scaling-up mindset \\ Ability to generate financial and technical resources to support the resource team and scale-up process
more generally \\ Compatibility with and understanding of the implementing organization \\ Understanding of the political, social, and cultural environments within which scale-up takes place \\ Technical, advocacy, management, and research/monitoring skills \\ Training capacity \\ Skills and experience with scaling-up and the use of data for decision making \\ Stability and a long-time horizon
}


include members with the skills necessary to fully support the implementing institution with the scale-up process, such as in advocacy, resource mobilization, management interventions, and others related to the attributes of success laid out in Table 1. Resource teams benefit when members hold senior, decision-making roles within institutions relevant to the scale-up process and have the time needed to devote to the demands of being a member.

In some countries where E2A was supporting scaleup, an existing group of key stakeholders (for example, a reproductive health technical working group) was mobilized to act as the resource team, while others were newly formed. These teams had the opportunity to learn about and apply the systematic approach to scale up supported by E2A, whether they were piloting implementation in ways that looked ahead to future expansion and institutionalization, developing a scale-up strategy, or managing a scale-up process.

In Cross River State, Nigeria, E2A mentored Pathfinder International country staff to help the state establish a new resource team composed of government, NGO, and civil society stakeholders to guide scale-up of a family planning service delivery-strengthening package (Oku, 2019). An early step was to build the resource team's capacity by teaching them about the scale-up framework and approach. This led directly to developing a scaling-up strategy for interventions that were successfully tested in operations research on task sharing by community health extension workers in family planning service delivery. Once the strategy was developed, the resource team worked over the next two years to guide state-level efforts to expand and institutionalize the task-shifting approach (Oku, 2019). Recognizing the value of a resource team to guide such a process, the then-incoming State Executive Secretary for Health requested the team to broaden their focus beyond family planning to support the scale-up of other health interventions in the state. Unfortunately, he was transferred to another ministry before the resource team's scope could be broadened. This illustrates a challenge that can arise when key resource team members, especially those from government, may leave their positions during scale-up.

In Uganda and Kenya, E2A supported multisectoral resource teams to scale up interventions tested in the initial pilot of the Health of People and Environment in the Lake Victoria Basin (HoPE-LVB) Project (Ghiron et al., 2014; Omimo et al., 2018). The HoPE-LVB Project had integrated sustainable development interventions from across multiple sectors including FP, other primary health-care services, and environmental conservation-a combination of synergistic interventions often referred to as the population, health, and environment approach (PHE). Because the piloted integration model was designed and implemented with scale-up in mind, with technical support from ExpandNet and E2A, it provided important global and local learning about how to successfully apply a scale-up mindset.

Influenced by the success of the cross-sectoral collaborations tested by HoPE-LVB in Uganda and Kenya, USAID gave funding to the East African Community's parastatal Lake Victoria Basin Commission to support their five member-nations to create or strengthen cross-sectoral national PHE networks and technical working groups. These in turn have served as resource teams to support scale-up of models of integrated development across the countries. This outcome was a result of HoPE-LVB's early and consistent engagement with both USAID and the Commission as part of the project's efforts to work with a scaling-up mindset.

While experiencing many successes, local resource teams also had difficulties. They rarely have all the attributes of success reflected in Table 1, and the E2A-supported resource teams were no exception. For example, in Cross River, Nigeria, the team lacked sufficient decision-making authority or available resources to act on some recommendations in the scaling-up strategy, such as institutionalizing health worker training capacity in every local government area (LGA) of the state (Oku, 2019). This meant that while scale-up was initiated during E2A's collaboration, the necessary training process was not completed, and considerable further resources were needed to train all health providers in each LGA. Fortunately, the state has since been able to mobilize World Bank resources to continue the training process. For HoPE-LVB, critical funding to sustain the project team could not be secured to ensure their continuity in providing the important technical support they had been giving to the national, cross-sectoral resource teams who were driving the scale-up process. Still, a sufficient level of capacity and political will had been built that scale-up continued even in their absence.

Other hurdles to successful scale-up can exist in the larger context, for example significant financial constraints, which can be nearly impossible to overcome. The Democratic Republic of the Congo (DRC) saw numerous scale-up successes associated with the work of a newly established, government-led, multi-stakeholder resource team who guided the process of instituting community-based access to family planning services in three project provinces (Mai et al., 2019). However, without sufficient governmental and donor support for expansion to other provinces, persistent financing constraints of the DRC health system may prove insurmountable in institutionalizing and expanding beyond the three initial provinces.

Working in weak and sometimes fragile public-sector systems can require making tradeoffs to sidestep poorly functioning systems and to instead employ parallel approaches. This is often at the cost of successful institutionalization, which diminishes chances for sustainability and ownership (Simmons et al., 2007; ExpandNet \& WHO, 2009, 2010, 
2011; Chandy \& Linn, 2011; Chandy et al., 2013). For example, it can be tempting to arrange for a regular supply of contraceptive commodities outside of the routine publicsector system when a project needs to ensure their availability at service delivery points. However, without a focus on strengthening the routine system, contraceptive stockouts will continue to hinder any scale-up effort and increased access to contraceptives will not be sustained in the future.

E2A and its local partners were repeatedly faced with making strategic decisions about such tradeoffs. Choosing how to proceed using a scaling-up mindset helped local teams to choose alternatives that would lead to greater sustainability whenever possible. Such balancing can be challenging, for example, when donors are looking for maximum short-term impact and results. In cases where a change is needed in the routine system to ensure increased access and better-quality service delivery, a strong argument can often be made for initially testing a new approach using special external resources or delivery channels. If the new approach is then shown to be valuable, advocacy at higher levels will be required to ensure that the routine system is strengthened sufficiently to allow for the new interventions to be implemented.

\section{Lesson 3: Sharing Scale-Up Learning Magnifies Results}

As is discussed in the ExpandNet/WHO guidance tools, a key determinant of scale-up success is the preparation and publication of careful documentation of experience. The important role of such work in driving further scale-up was clearly demonstrated by E2A. For example, documentation and dissemination of E2A experience in the DRC with expanding access to community-based FP through outreach from health facilities influenced national strategic plans and operational guidelines (Mai et al., 2019). Similarly, in Niger, the documentation from efforts to establish adolescent and youth-friendly $\mathrm{FP} / \mathrm{RH}$ services in university and communitybased settings also led to national-level institutionalization of the tested approach (Benevides et al., 2019).

While E2A, with ExpandNet as a partner, was learning how best to support scale-up processes in countries, other technical agencies were also engaged in supporting similar initiatives. Given its global technical leadership role and scale-up mandate, E2A worked to disseminate its learning in a wide range of international venues and publications. A strategic decision was made in 2013 to establish and lead a global Community of Practice (CoP) on Systematic Approaches to Scale-up in FP/RH, headquartered in Washington DC (E2A, 2020), whose work still continues today. The CoP provided an early and ongoing platform for sharing experience among representatives of a wide range of technical and donor institutions who were engaged in, had expertise or an interest in the subject of scale-up. Assembling this type of group enabled E2A to learn from others engaged in scale-up work across health and development interventions. Over the years, the quarterly meetings also facilitated exploration of related technical issues such as sustainability and adaptation as well as more general exchange with allied fields such as implementation science.

The CoP's learning exchange enriched the global dialog around scale-up and amplified attention to the need for a strong focus on scaling among technical agencies and donors. Participants in the quarterly meetings and technical convenings benefited greatly from discussions about fundamental scale-up considerations and from exposure to the field experience shared by visitors. For example, participation by the leader of the Nigerian NGO MidSpace in a quarterly $\mathrm{CoP}$ meeting provided an opportunity to learn about their perspective on the importance of country and government leadership in scaling up and innovative ways of supporting such processes. Moreover, other presentations by E2A collaborators from Kenya, Niger, Nigeria and Uganda illustrated how capacity was developed locally and how mindsets shifted to a scale-up perspective in-country.

In 2015, colleagues from Management Systems International and the Brookings Institution decided to start a scaling-up CoP focused on global health and other development issues including education, agriculture, rural development, social enterprises and more (Community of Practice on Scaling up Development Outcomes, 2021). Collaboration with this CoP has provided the E2A-led CoP opportunities to interact with a multisectoral group of stakeholders about the frontiers of understanding scale-up.

\section{Conclusions}

Applying a scale-up mindset is essential to achieving sustainable expansion and institutionalization of successfully tested interventions. A key learning from the E2A experience is that shifting from a narrow project approach to one focused on system strengthening does not happen rapidly. The project benefited from having an explicit mandate to focus on scale-up, but it took varying amounts of time and support for the technical team members and country collaborators to internalize and apply the new mindset. This in turn influenced the extent to which E2A-supported country-level activities were pursued with a systematic scale-up focus.

This learning has important implications for what can be achieved during a project's time horizon. Given that it is often required to orient project team members to a scaling-up mindset and then to build scale-up capacity among the staff and the country teams with whom they collaborate, implementing scale-up takes time. For example, even a five- or seven-year project may result only in 
initial scale-up progress, and therefore, steps to ensure a full transition to government or other stakeholders are needed prior to a project's end. A key component of transition planning must be ensuring government ownership and the existence of a well-positioned and adequately funded and skilled resource team who can continue to guide the scale-up process.

Moreover, a scaling mindset must be adopted not only by project leaders but by donors as well. Some donors have already been making this shift, but reorientation among others is still required. Given that scale-up is a complex task that takes time, perseverance and new ways of working, the more that donors adopt this emphasis, the more likely it will be that lasting benefits can be achieved.

E2A, and through it, USAID made a significant contribution to building in-country capacity for applying systematic approaches to scale up among stakeholders with whom the project worked. In addition, E2A influenced others at the global level to focus greater attention on scaleup and to move towards the necessary mindset shift. The project has illustrated how donor-funded projects can and should focus systematically on scale-up from the outset, how they can appropriately ensure the development of scale-up strategies, and how to support strategic management of scale-up processes. As the E2A project draws to a close, it will leave the legacy of having advanced the scaling-up discourse and built the capacity of scaling-up resource professionals around the world. However, the project's many partners and allies must continue to advance this work in the future so as to reach more people with the health and development interventions they need.

Acknowledgements The authors gratefully acknowledge the dedication of members of the Evidence to Action technical team, Pathfinder International, and in-country partners in Burkina Faso, Cote d'Ivoire, the Democratic Republic of the Congo, Kenya, Niger, Nigeria, Senegal, Uganda, and other countries whose determination to apply the ExpandNet/WHO systematic approach to scale up highlighted here led to productive outcomes. Special thanks also go to Shae Langley and Zevik Citron for editorial support. This article and the work highlighted therein were supported through a cooperative agreement from the United States Agency for International Development under Award No. AID-OAA-A-11- 00024 (Evidence to Action). The opinions expressed here are those of the authors and do not necessarily reflect the views of Pathfinder International or the U.S. Agency for International Development.

Author Contributions LG and RS conceptualized and drafted the initial and subsequent drafts, with support from PF. RBa and ERF provided periodic technical input and support. RBe and AN were instrumental in supporting implementation at the country levels for the work discussed in the paper. All authors endorsed and approved the manuscript.

\section{Declarations}

Conflict of interest There are no conflict of interest to declare.
Open Access This article is licensed under a Creative Commons Attribution 4.0 International License, which permits use, sharing, adaptation, distribution and reproduction in any medium or format, as long as you give appropriate credit to the original author(s) and the source, provide a link to the Creative Commons licence, and indicate if changes were made. The images or other third party material in this article are included in the article's Creative Commons licence, unless indicated otherwise in a credit line to the material. If material is not included in the article's Creative Commons licence and your intended use is not permitted by statutory regulation or exceeds the permitted use, you will need to obtain permission directly from the copyright holder. To view a copy of this licence, visit http://creativecommons.org/licenses/by/4.0/.

\section{References}

Aichatou, B., Seck, C., Anne, T. S. B., Deguenovo, G. C., Ntabona, A., \& Simmons, R. (2016). Strengthening government leadership in family planning programming in Senegal: From proof of concept to proof of implementation in 2 Districts. Global Health: Science and Practice, 4(4), 568-581. https://doi.org/10.9745/ GHSP-D-16-00250

Barker, P. M., Reid, A., \& Schall, M. W. (2016). A framework for scaling up health interventions: Lessons from large-scale improvement initiatives in Africa. Implementation Science. https://doi.org/ 10.1186/s13012-016-0374-x

Benevides, R., Chau, K., Ousseini, A., Innocent, I., \& Simmons, R. (2019). Engaging students to improve sexual and reproductive health: A report of the University Leadership for Change initiative in Niger. African Journal of Reproductive Health, 23(1):55-64. https://pubmed.ncbi.nlm.nih.gov/31034172/

Bulthuis, S. E., Kok, M. C., Raven, J., \& Dieleman, M. A. (2020). Factors influencing the scale-up of public health interventions in low- and middle-income countries: A qualitative systematic literature review. Health Policy and Planning, 35, 219-234. https:// doi.org/10.1093/heapol/czz140

Chandy, L., Hosono, A., Kharas, H., \& Linn, J. F. (2013). Getting to scale: How to bring development solutions to millions of poor people. Brookings Institution Press. https://www.brookings.edu/ book/getting-to-scale/

Chandy, L., \& Linn, J. F. (2011). Taking development activities to scale in fragile and low capacity environments. Washington, DC: Brookings Institution, Global Economy and Development. https://www.brookings.edu/wp-content/uploads/2016/06/Scali ng-Up-Fragile-States.pdf

Community of Practice on Scaling up Development Outcomes. (2021). https://www.scalingcommunityofpractice.com

Cooley, L., \& Linn, J. (2014). Taking innovations to scale: Methods, applications and lessons. Washington, DC: Results for Development Institute. https://www.usaid.gov/sites/default/files/docum ents/1865/v5web_R4D_MSI-BrookingsSynthPaper0914-3.pdf

E2A-led Community of Practice on Systematic Approaches to Scaleup. (2020). https://www.e2aproject.org/about-us/cop/

Evidence to Action (E2A) Project. (2020). https://www.e2aproject.org/ ExpandNet \& World Health Organization. (2009). Practical guidance for scaling up health service innovations. Geneva: World Health Organization. https://www.who.int/reproductivehealth/publicatio ns/strategic_approach/9789241598521/en/ and http://www.expan dnet.net/tools.htm

ExpandNet \& World Health Organization. (2010). Nine steps for developing a scaling-up strategy. Geneva: World Health Organization. http://www.who.int/reproductivehealth/publications/strategic approach/9789241500319/en/index.html and http://www.expan dnet.net/tools.htm 
ExpandNet \& World Health Organization. (2011). Beginning with the end in mind: Planning pilot projects and other programmatic research for successful scaling up. Geneva: World Health Organization. http://www.expandnet.net/tools.htm and https:// www.who.int/reproductivehealth/publications/strategic_appro ach/9789241502320/en/

ExpandNet website. (2021). https://expandnet.net/

Fajans, P., \& Simmons, R. (2016). The Urban Reproductive Health Initiatives: A comparative review of ISSU, NURHI, Tupange and the UHI. ExpandNet report submitted to the Bill and Melinda Gates Foundation. https://expandnet.net/PDFs/URHI\%20Com parative $\% 20$ Review\%20of\%20ISSU\%20NURHI\%20Tupange\% 20and\%20UHI.pdf

Ghiron, L., Shillingi, L., Kabiswa, C., Ogonda, G., Omimo, A., Ntabona, A., Simmons, R., \& Fajans, P. (2014). Beginning with sustainable scale up in mind: initial results from a population, health and environment project in East Africa. Reproductive Health Matters, 22(43), 84-92. https://doi.org/10.1016/s0968-8080(14) 43761-3

Hartmann, A., \& Linn, J. F. (2008). Scaling up: A framework and lessons for development effectiveness from literature and practice. Wolfensohn Center for Development, Working Paper 5. Washington, DC: The Brookings Institution. https://doi.org/https://doi.org/ $10.2139 /$ ssrn. 1301625

Igras, S., Sinai, I., Mukabatsinda, M., Ngabo, F., Jennings, V., \& Lundgren, R. (2014). Systems approach to monitoring and evaluation guides scale up of the Standard Days Method of family planning in Rwanda. Global Health: Science and Practice, 2(2), 234-244. https://doi.org/10.9745/GHSP-D-13-00165

Keyonzo, N., Nyachae, P., Kagwe, P., Kilonzo, M., Mumba, F., Owino, K., Kichamu, G., Kigen, B., Fajans, P., Ghiron, L., \& Simmons, R. (2015). From project to program: Tupange's experience with scaling up family planning interventions in urban Kenya. Reproductive Health Matters, 23(45), 103-113. https://doi.org/10.1016/j. rhm.2015.06.010

Mai, M., Hassen, E., Ntabona, A. B., Bapura, J., Sarathy, M., Yodi, R., \& Mujani, Z. (2019). Government ownership and adaptation in scale-up: experiences from community-based family planning programme in the Democratic Republic of the Congo. African Journal of Reproductive Health, 23(4):35-45 https://pubmed.ncbi. nlm.nih.gov/32227738/

Management Systems International. (2016). Scaling up - from vision to large-scale change: Tools and techniques for practitioners, second edition. Washington, D.C.: Management Systems International. https://msiworldwide.com/sites/default/files/additional-resources/ 2018-11/ScalingUp_3rdEdition.pdf

Milat, A. J., Bauman, A., \& Redman, S. (2015). Narrative review of models and success factors for scaling up public health interventions. Implementation Science, 10(1), 113. https://doi. org/10.1186/s13012-015-0301-6

Milat, A. J., Newson, R., King, L., Rissel, C., Wolfenden, L., Bauman, A., Redman, S., \& Giffin, M. (2016). A guide to scaling up population health interventions. Public Health Research \& Practice, 26(1), e2611604. https://doi.org/https://doi.org/10.17061/phrp2 611604

Oku, A. (2019). Lessons learned from the systematic scale up of family planning task-shifting and task-sharing in Cross River State, Nigeria. Washington, DC: Evidence to Action (E2A) Project. https://www.e2aproject.org/wp-content/uploads/CRS-FP-Scaleup-TSTS-Documentation-FINAL.pdf

Omimo, A., Taranta, D., Ghiron, L., Kabiswa, C., Aibe, S., Kodande, M., Nalwoga, C., Mugaya, S., \& Onduso, P. (2018). Applying ExpandNet's systematic approach to scaling up in an integrated population, health and environment project in East Africa. Social Sciences, 7(2), 8. https://doi.org/10.3390/socsci7010008

Simmons, R., Fajans, P., \& Ghiron, L. (2007). Scaling up health service delivery: From pilot innovations to policies and programmes. Geneva: World Health Organization. http://www.who.int/repro ductivehealth/publications/strategic_approach/9789241563512/ en/index.html and http://www.expandnet.net/tools.htm

Spicer, N., Bhattacharya, D., Dimka, R., Fanta, F., Mangham-Jefferies, L., Schellenberg, J., Tamire-Woldemariam, A., Walt, G., \& Wickremasinghe, D. (2014). 'Scaling-up is a craft not a science': Catalysing scale-up of health innovations in Ethiopia, India and Nigeria. Social Science \& Medicine, 121, 30-38. https://doi.org/ 10.1016/j.socscimed.2014.09.046

Spicer, N., Hamza, Y. A., Berhanu, D., Gautham, M., Schellenberg, J., Tadesse, F., Umar, N., \& Wickremasinghe, D. (2018). “The development sector is a graveyard of pilot projects!" Six critical actions for externally funded implementers to foster scale-up of maternal and newborn health innovations in low and middleincome countries. Globalization and Health, 14(1), 74. https:// doi.org/10.1186/s12992-018-0389-y

United Nations. (2015). Transforming our World: The 2030 Agenda for Sustainable Development. United Nations A/RES/70/1. https:// sustainabledevelopment.un.org/post2015/transformingourworld/ publication

Wickremasinghe, D., Gautham, M., Umar, N., Berhanu, D., Schellenberg, J., \& Spicer, N. (2018). "It's about the idea hitting the bull's eye": How aid effectiveness can catalyse the scale-up of health innovations. International Journal of Health Policy and Management, 7(8), 718-727. https://doi.org/https://doi.org/10. 15171/ijhpm.2018.08

Yamey, G. (2011). Scaling up global health interventions: A proposed framework for success. PLoS Medicine, 8(6), e1001049. https:// doi.org/10.1371/journal.pmed.1001049 